TRANSACTIONS OF THE

AMERICAN MATHEMATICAL SOCIETY

Volume 353, Number 7, Pages 2879-2895

S 0002-9947(01)02708-8

Article electronically published on March 12, 2001

\title{
LIVŠIC THEOREMS FOR CONNECTED LIE GROUPS
}

\author{
M. POLLICOTT AND C. P. WALKDEN
}

\begin{abstract}
Let $\phi$ be a hyperbolic diffeomorphism on a basic set $\Lambda$ and let $G$ be a connected Lie group. Let $f: \Lambda \rightarrow G$ be Hölder. Assuming that $f$ satisfies a natural partial hyperbolicity assumption, we show that if $u: \Lambda \rightarrow G$ is a measurable solution to $f=u \phi \cdot u^{-1}$ a.e., then $u$ must in fact be Hölder. Under an additional centre bunching condition on $f$, we show that if $f$ assigns 'weight' equal to the identity to each periodic orbit of $\phi$, then $f=u \phi \cdot u^{-1}$ for some Hölder $u$. These results extend well-known theorems due to Livšic when $G$ is compact or abelian.
\end{abstract}

\section{INTRODUCTION}

Let $\phi$ be a $C^{1}$ diffeomorphism of a compact Riemannian manifold $M$. If we can restrict $\phi$ to a (locally maximal) hyperbolic set $\Lambda$, then we call $\phi: \Lambda \rightarrow \Lambda$ a hyperbolic map. A familiar example is a (transitive) Anosov diffeomorphism, in this case $\Lambda=M$. More generally, an Axiom $A$ diffeomorphism restricted to a basic set in the non-wandering set is hyperbolic. We give a complete definition in $\$ 2$.

Let $f: \Lambda \rightarrow \mathbb{R}$ be Hölder. It is often important to determine when $f$ is a coboundary, i.e. $f=u \phi-u$ for some Hölder $u: \Lambda \rightarrow \mathbb{R}$. This was first studied by Livšic [L1, L2] and, in more general circumstances, has been the subject of several recent papers [NP, [NT, [PP2] [P] $[\mathrm{Sc}, \mathrm{W} 1, \mathrm{~W} 2]$.

Let $m$ be the Gibbs measure corresponding to a Hölder continuous function (see \$2). In [L2], Livšic showed that, for any Hölder $f: \Lambda \rightarrow \mathbb{R}$, a measurable solution $u: \Lambda \rightarrow \mathbb{R}$ to the coboundary equation $f=u \phi-u$ a.e. has a Hölder continuous version, i.e. $u$ is necessarily equal a.e. to a Hölder function $u^{\prime}$ for which $f=u^{\prime} \phi-u^{\prime}$ everywhere.

It is natural to try to generalise this result by replacing $\mathbb{R}$ with an arbitrary connected Lie group $G$. More specifically, we ask for which connected Lie groups and Hölder $f$ does the following property hold:

Property (R) (Regularity). If $f: \Lambda \rightarrow G$ is Hölder and $u$ is a measurable solution to the coboundary equation

$$
f=u \phi \cdot u^{-1} \text { a.e. }
$$

then $u$ has a Hölder continuous version.

Livšic [L2] proved that Property (R) holds for the case $G=\mathbb{R}$. In fact, the proof given there works for any connected Lie group with a bi-invariant metric. (Moreover, for the more general equation $f(x)=u(\phi x) g(x) u(x)^{-1}$ and compact $G$, the analogue of Property (R) was proved in PP2. More generally, Schmidt showed

Received by the editors January 31, 1999 and, in revised form, April 12, 2000.

2000 Mathematics Subject Classification. Primary 58F11; Secondary 58F15.

(C)2001 American Mathematical Society 
that the analogue of Property (R) holds when $f$ and $g$ satisfy a notion of 'bounded distortion' $[\mathrm{Sc}$; see 99.) One of our aims in this note is to extend Property (R) to a larger class of Hölder functions $f$.

In order to state our main theorems, we need to make some definitions.

The hyperbolicity of $\left.\phi\right|_{\Lambda}$ can be characterised in terms of the Mather spectrum of $\phi$. Let $\mathcal{X}(\Lambda)$ denote the Banach space of continuous vector fields on $\Lambda$. Define $\phi_{*}: \mathcal{X}(\Lambda) \rightarrow \mathcal{X}(\Lambda)$ by $\left(\phi_{*} v\right)(x)=d \phi\left(v\left(\phi^{-1} x\right)\right)$. The Mather spectrum $\operatorname{spec}(\phi)$ of $\phi$ is the spectrum of $\phi_{*}$ acting on the complexification of $\mathcal{X}(\Lambda)$.

Let $G$ be a connected Lie group with Lie algebra $L(G)$. Recall that the adjoint map Ad : $G \rightarrow \operatorname{Aut}(L(G))$ is defined so that $\operatorname{Ad}(g)$ is the derivative of conjugation by $g$. We introduce the following:

Hypothesis (PH) (Partial Hyperbolicity). Let $\phi: \Lambda \rightarrow \Lambda$ be hyperbolic with Mather spectrum contained in the annuli $\left\{z|0<| z \mid<\lambda_{s}\right\},\left\{z \mid \lambda_{u}<\right.$ $|z|<\infty\}$, for some $\lambda_{s}<1<\lambda_{u}$. We say that a Hölder continuous $f: \Lambda \rightarrow G$ satisfies Hypothesis (PH) if $\lambda_{s}<\mu_{s} \leq 1 \leq \mu_{u}<\lambda_{u}$ where

$$
\begin{aligned}
& \mu_{s}=\lim _{n \rightarrow \infty}\left(\sup _{x \in \Lambda}\left\|\operatorname{Ad}\left(f\left(\phi^{n-1} x\right) \cdots f(\phi x) f(x)\right)\right\|\right)^{1 / n}, \\
& \mu_{u}=\lim _{n \rightarrow \infty}\left(\sup _{x \in \Lambda}\left\|\operatorname{Ad}\left(f\left(\phi^{n-1} x\right) \cdots f(\phi x) f(x)\right)^{-1}\right\|\right)^{-1 / n} .
\end{aligned}
$$

For example, if $G$ is compact, nilpotent or soluble of type $R$, then both of the above limits are equal to 1 . If $f$ satisfies the notion of bounded distortion as defined in Sc], then $\mu_{s}=1=\mu_{u}$.

The terminology is motivated by the study of partially hyperbolic dynamics. If $\phi$ is a $C^{2}$ Anosov diffeomorphism and $f: \Lambda \rightarrow G$ is a $C^{2}$ function satisfying Hypothesis $(\mathrm{PH})$, then the skew product $(x, y \Gamma) \mapsto(\phi(x), f(x) y \Gamma)$ with a compact homogeneous space $G / \Gamma$ as fibre is partially hyperbolic in the sense of $\mathrm{BP}$.

For $\theta \in(0,1)$ let

$$
C^{\theta}(\Lambda, G)=\left\{f: \Lambda \rightarrow G \mid \sup _{x \neq x^{\prime}} \rho\left(f(x), f\left(x^{\prime}\right)\right) / d\left(x, x^{\prime}\right)^{\theta}<\infty\right\}
$$

denote the space of Hölder functions $\Lambda \rightarrow G$ with Hölder exponent $\theta$. We shall be interested in Hölder functions with a sufficiently large Hölder exponent. Define

$$
\theta_{\mathrm{PH}}=\max \left\{\log \mu_{s} / \log \lambda_{s}, \log \lambda_{u} / \log \mu_{u}\right\}
$$

and observe that if $f$ satisfies Hypothesis $(\mathrm{PH})$, then $\theta_{\mathrm{PH}}<1$.

In this paper we shall establish the following.

Theorem A. Let $\phi: \Lambda \rightarrow \Lambda$ be a hyperbolic diffeomorphism equipped with a Hölder equilibrium state. Let $G$ be a connected Lie group. Suppose $f \in C^{\theta}(\Lambda, G), \theta>\theta_{\mathrm{PH}}$, satisfies Hypothesis (PH). Then Property $(R)$ holds.

It is clear that there are obstructions to $f: \Lambda \rightarrow G$ being a coboundary, namely that $f$ must assign 'weight' equal to the identity to each periodic point of $\phi$. More specifically, if $\phi^{n} x=x$, then $f^{n}(x)=f\left(\phi^{n-1} x\right) \cdots f(x)=u\left(\phi^{n} x\right) \cdot u(x)^{-1}=e$, the group identity. In [L1], Livšic proved the converse of this observation when $G$ admits a bi-invariant metric: if $f$ is Hölder and $f^{n}(x)=e$ whenever $\phi^{n} x=x$, then $f=u \phi \cdot u^{-1}$ for some Hölder $u$. We can ask for which class of connected Lie groups and Hölder functions does the following property hold: 
Property (PO) (Periodic Obstructions). If $f: \Lambda \rightarrow G$ is Hölder and $f^{n}(x)=$ $e$, the group identity, whenever $\phi^{n} x=x$, then there exists a Hölder solution $u: \Lambda \rightarrow G$ to the coboundary equation $f=u \phi \cdot u^{-1}$.

We shall need the following hypothesis:

Hypothesis (CB) (Centre Bunching). Let $\lambda_{s}, \lambda_{u}, \mu_{s}, \mu_{u}$ be as above. We say that a Hölder continuous function $f: \Lambda \rightarrow G$ satisfies Hypothesis (CB) if it satisfies Hypothesis (PH) and, in addition, $\lambda_{s}<\mu_{u}^{-1}$ and $\mu_{s}^{-1}<\lambda_{u}$.

This terminology is also motivated by the study of partially hyperbolic dynamics. Usually, centre bunching is interpreted to mean that $\mu_{s} \mu_{u}$ is close to 1. Clearly, if $\mu_{s} \mu_{u}$ is close to 1 , then Hypothesis (CB) holds.

We again need the Hölder exponent of $f$ to be sufficiently large. Define

$$
\theta_{\mathrm{CB}}=\max \left\{\theta_{\mathrm{PH}}, \log \mu_{s}^{-1} / \log \lambda_{u}, \log \mu_{u} / \log \lambda_{s}^{-1}\right\}
$$

and observe that Hypothesis $(\mathrm{CB})$ implies $\theta_{C B}<1$.

We prove the following:

Theorem B. Let $\phi: \Lambda \rightarrow \Lambda$ be a hyperbolic diffeomorphism and let $G$ be a connected Lie group. Suppose $f \in C^{\theta}(\Lambda, G), \theta>\theta_{\mathrm{CB}}$, satisfies Hypothesis $(C B)$. Then Property (PO) holds.

Observe that the hypotheses of Theorem $\mathrm{B}$ are more restrictive than the hypotheses of Theorem $\mathrm{A}$

Livšic [L1] proved that Property (PO) holds when $G$ admits a bi-invariant metric. Property (PO) is discussed for the more general equation $f(x)=u(\phi x) g(x) u(x)^{-1}$ in $[\mathrm{P}]$ (in the compact case) and in $[\mathrm{Sc}$ (when $f, g$ satisfy a 'bounded distortion' condition).

We shall study Properties (PO) and (R) by looking separately at soluble and non-soluble Lie groups. Indeed, we shall see that Hypotheses $(\mathrm{PH})$ and $(\mathrm{CB})$ are not needed when $G$ is soluble (see 93 ).

Recall that the radical $R=\operatorname{Rad} G$ of a connected Lie group $G$ is the largest connected normal soluble Lie subgroup of $G$ and let $\pi_{R}: G \rightarrow G / R$ denote the quotient map. The Lie group $G / R$ is semi-simple. We can weaken the hypotheses of Theorems $\mathrm{A}$ and $\mathrm{B}$ by only assuming that $\pi_{R} f: \Lambda \rightarrow G / R$ satisfy Hypotheses $(\mathrm{PH})$ and $(\mathrm{CB})$, respectively. These results are stated below as Theorems [7.2, 7.3

We also prove analogues of the above results for shifts of finite type (Theorems 7.4, 7.5) and for hyperbolic flows (Theorems 8.1, 8.2).

This note is organised as follows. In $₫ 2$ we discuss some background on hyperbolic dynamics and, in particular, how to model them by shifts of finite type. In 3 we begin our analysis in the technically simpler case of a soluble Lie group and a shift of finite type. Following some technical results in 4 , we prove Theorem $\mathrm{A}$ (in \$5) and Theorem B (in $\$ 6$ ). In $\$ 7$ we prove Theorems 7.2 and 7.3 , the strongest versions of our results.

Acknowledgements. We would like to thank Matt Nicol and Richard Sharp for useful conversations. MP was partially supported by the Leverhulme Trust and CPW was supported by the EPSRC.

\section{HyPERBoliC DIFFEOMORPHISMS AND SYMBOLIC DYNAMICS}

We say that a $C^{1}$ diffeomorphism $\phi$ on a compact manifold $M$ restricted to a $\phi$-invariant subset $\Lambda$ is hyperbolic if $\operatorname{spec}(\phi)$ is contained in the disjoint union of 
two annuli $\left\{z|0<| z \mid<\lambda_{s}\right\} \cup\left\{z\left|\lambda_{u}<\right| z \mid<\infty\right\}$ for some $0<\lambda_{s}<1<\lambda_{u}<\infty$ and, in addition, there exists a dense orbit, the periodic points in $\Lambda$ are dense in $\Lambda$ and $\Lambda$ is locally maximal, i.e. $\Lambda=\bigcap_{n=-\infty}^{\infty} \phi^{-n} U$ for some open neighbourhood $U \supset \Lambda$. Examples of hyperbolic maps include (transitive) Anosov diffeomorphisms and Axiom $A$ diffeomorphisms restricted to a basic set.

Let $g: \Lambda \rightarrow \mathbb{R}$ be Hölder. Consider the quantity $P(g)=\sup _{\mu}\left\{h_{\mu}(\phi)+\int g d \mu\right\}$ as $\mu$ ranges over all $\phi$-invariant probabilities. We call $P(g)$ the pressure of $g$. This supremum is achieved by a unique invariant measure $m[\mathrm{~B}$, which we call the (Hölder) equilibrium state or Gibbs measure corresponding to $g$.

Let $\phi$ be hyperbolic and let $d$ be a metric on $\Lambda$. We define the stable manifold $W^{s}(x)$ through $x \in \Lambda$ to be

$$
W^{s}(x)=\left\{x^{\prime} \in M \mid d\left(\phi^{n} x, \phi^{n} x^{\prime}\right) \rightarrow 0 \text { as } n \rightarrow \infty\right\} .
$$

These are $C^{1}$ immersed submanifolds and define a Hölder continuous lamination of $\Lambda$. Similarly, we can define the unstable manifold $W^{u}(x)$ :

$$
W^{u}(x)=\left\{x^{\prime} \in M \mid d\left(\phi^{-n} x, \phi^{-n} x^{\prime}\right) \rightarrow 0 \text { as } n \rightarrow \infty\right\} .
$$

For $\varepsilon>0$ sufficiently small, we define the local stable and local unstable manifolds by

$$
W_{\varepsilon}^{s}(x)=\left\{x^{\prime} \in M \mid d\left(\phi^{n} x, \phi^{n} x^{\prime}\right) \leq \varepsilon \text { for all } n \geq 0\right\}
$$

and

$$
W_{\varepsilon}^{u}(x)=\left\{x^{\prime} \in M \mid d\left(\phi^{-n} x, \phi^{-n} x^{\prime}\right) \leq \varepsilon \text { for all } n \geq 0\right\} ;
$$

these are subsets of $W^{s}(x), W^{u}(x)$, respectively. If $x$ and $y$ are sufficiently close, then the set $W_{\varepsilon}^{u}(x) \cap W_{\varepsilon}^{s}(y)$ consists of a single point and this point lies in $\Lambda$. We denote this point by $\langle x, y\rangle$.

We have the following characterisation of stable manifolds ([KH] for example): $y \in W^{s}(x)$ precisely when $d\left(\phi^{n} x, \phi^{n} y\right) \leq C \lambda_{s}^{n} d(x, y)$, for some constant $C>0$ independent of $n$. If $y \in W_{\varepsilon}^{s}(x)$, then the constant can be chosen independently of $x, y$. A similar criterion exists for unstable manifolds.

Let $x \in \Lambda$. We define the homoclinic set $W(x)$ through $x$ to be

$$
W(x)=W^{s}(x) \cap W^{u}(x)=\left\{x^{\prime} \in M \mid d\left(\phi^{n} x, \phi^{n} x^{\prime}\right) \rightarrow 0 \text { as }|n| \rightarrow \infty\right\} .
$$

Then for each $x, W(x) \cap \Lambda$ is dense in $\Lambda$ B].

We shall also need the following well-known result.

Theorem 2.1 (Anosov's closing lemma $[\mathrm{A}]$ ). There exists $\delta>0$ such that if $x \in \Lambda$ satisfies $d\left(\phi^{n} x, x\right)<\delta$, then there exists a periodic point $w=\phi^{n} w \in \Lambda$ such that, for $j=0, \ldots, n-1$, we have

$$
d\left(\phi^{j} x, \phi^{j} w\right) \leq C\left(\max \left\{\lambda_{s}, \lambda_{u}^{-1}\right\}\right)^{\min \{j, n-j\}} d\left(\phi^{n} x, x\right),
$$

for some constant $C$ independent of $x$.

2.1. Symbolic dynamics for hyperbolic diffeomorphisms. Let $A$ be a $k \times k$ a periodic matrix with entries from $\{0,1\}$. We define the space

$$
\Sigma=\left\{x=\left(x_{n}\right)_{n=-\infty}^{\infty} \mid A_{x_{n} x_{n+1}}=1 \text { for each } n \in \mathbb{Z}\right\} .
$$

For each $\theta \in(0,1)$ we define a metric $d^{\theta}$ by $d^{\theta}(x, y)=\theta^{n(x, y)}$ where $n(x, y)=$ $\sup \left\{n \mid x_{i}=y_{i}\right.$ for all $\left.|i| \leq n\right\}$ if $x \neq y$. If $f: \Sigma \rightarrow Y$ for some metric space $Y$ is 
Lipschitz continuous with respect to $d^{\theta}$, then we shall abuse terminology and say that $f$ is Hölder of exponent $\theta$. Denote the space of such functions by $F_{\theta}(\Sigma, Y)$.

Define the shift map $\sigma: \Sigma \rightarrow \Sigma$ by $(\sigma x)_{n}=x_{n+1}$. The map $\sigma$ is a homeomorphism of the compact zero-dimensional space $\Sigma$. We call $\sigma: \Sigma \rightarrow \Sigma$ a shift of finite type.

Let $g: \Sigma \rightarrow \mathbb{R}$ be Hölder and consider the quantity $h_{\mu}(\sigma)+\int g d \mu$ as $\mu$ ranges over all $\sigma$-invariant probability measures. This is maximised by a unique $\sigma$-invariant measure $[\mathrm{B}]$ which we call the equilibrium state of $g$ and denoted by $m$. We call $m$ a Hölder equilibrium state.

By a slight abuse of notation, we write $W^{s}(x)=\left\{y \in \Sigma \mid y_{i}=x_{i}\right.$ for all sufficiently large $i\}$ and $W_{\varepsilon}^{s}(x)=\left\{y \in \Sigma \mid y_{i}=x_{i}\right.$ for all $\left.i \geq 0\right\}$ and refer to these as the stable and local stable manifolds through $x$. Similarly we can define $W^{u}(x)$ and $W_{\varepsilon}^{u}(x)$.

A hyperbolic diffeomorphism can be represented as a shift of finite type via a Markov partition, and this is the key to proving our main results.

Proposition $2.2([\mathrm{~B}])$. Let $\phi: \Lambda \rightarrow \Lambda$ by hyperbolic. Then there exists a shift of finite type $\sigma: \Sigma \rightarrow \Sigma$ and a Hölder continuous map $\pi: \Sigma \rightarrow \Lambda$ such that

1. $\pi \sigma=\phi \pi$,

2. if $m$ is a Hölder equilibrium state for $\phi$, then $m=\pi_{*} \tilde{m}$ for some Hölder equilibrium state $\tilde{m}$ for $\sigma$,

3. $\pi$ is one-to-one a.e. for any Hölder equilibrium state.

2.2. Local product structure of equilibrium states. Let $\phi$ be hyperbolic. If $\varepsilon$ is sufficiently small and $x, x^{\prime} \in \Lambda$ are sufficiently close, then we have the holonomy map $\rho_{x, x^{\prime}}: W_{\varepsilon}^{u}(x) \rightarrow W_{\varepsilon}^{u}\left(x^{\prime}\right)$ defined by sliding along the local stable manifolds. That is, $\rho_{x, x^{\prime}}(y)$ is the unique point contained in $W_{\varepsilon}^{u}\left(x^{\prime}\right) \cap W_{\varepsilon}^{s}(y)$. We denote this point by $\left\langle x^{\prime}, y\right\rangle$.

For a shift of finite type, this holonomy map takes a particularly simple form. If $x, y \in \Sigma$ are sufficiently close so that $x_{0}=y_{0}$, then we define the point $\langle x, y\rangle \in \Lambda$ by $(\langle x, y\rangle)_{n}=x_{n}$ for $n \leq 0$ and $(\langle x, y\rangle)_{n}=y_{n}$ for $n \geq 0$. The holonomy map $\rho_{x, x^{\prime}}: W_{\varepsilon}^{u}(x) \rightarrow W_{\varepsilon}^{u}\left(x^{\prime}\right)$ is then given by $\rho_{x, x^{\prime}}(y)=\left\langle x^{\prime}, y\right\rangle$.

We shall prove Theorem $\mathrm{A}$ by first proving the existence of a Hölder solution along stable and unstable manifolds. To do this, we need to be able to interpret (1) on stable and unstable manifolds. That this is possible follows from the following natural result. Recall that two measures are equivalent if they have the same sets of measure zero. To show two measures are equivalent it is sufficient to show that their Radon-Nikodym derivative is bounded away from zero and infinity.

Proposition 2.3. Let $m$ be a Hölder equilibrium state for $\phi$ corresponding to the function $g: \Lambda \rightarrow \mathbb{R}$. Then there exists a family of measures $\left\{m_{x}^{u}\right\}$ with $m_{x}^{u}$ supported on $W^{u}(x)$ such that

1. $\phi^{*} m_{x}^{u}$ and $m_{\phi x}^{u}$ are equivalent; indeed, $d \phi^{*} m_{x}^{u}=e^{g(\phi x)-P(g)} d m_{\phi x}^{u}$,

2. For each $x, x^{\prime}$ sufficiently close, $\rho_{x, x^{\prime}}^{*} m_{x}^{u}$ and $m_{x^{\prime}}^{u}$ are equivalent; indeed, $d \rho_{x, x^{\prime}}^{*} m_{x}^{u}=e^{\omega_{x, x^{\prime}}(\cdot)} d m_{x^{\prime}}^{u}$ where

$$
\omega_{x, x^{\prime}}(y)=\sum_{j=0}^{\infty} g\left(\phi^{j}\langle x, y\rangle\right)-g\left(\phi^{j}\left\langle x^{\prime}, y\right\rangle\right) .
$$

There is a family $\left\{m_{x}^{s}\right\}$ of measures supported on $W^{s}(x)$ satisfying similar properties. 
The measure $m$ is locally equivalent to the product $m_{x}^{s} \times m_{x}^{u}$.

In other words, the stable and unstable laminations are absolutely continuous with respect to the measures on stable and unstable manifolds induced from the Gibbs measure. We shall use Proposition 2.3 in the following way. If $N \subset \Lambda$ has $m$-measure zero, then $m_{x}^{u}\left(N \cap W_{\varepsilon}^{u}(x)\right)=0$ and $m_{x}^{s}\left(N \cap W_{\varepsilon}^{s}(x)\right)=0$ for $m$-a.e. $x \in \Lambda$. Conversely, if $m_{x}^{u}\left(N \cap W_{\varepsilon}^{u}(x)\right)=0$ and $m_{x}^{s}\left(N \cap W_{\varepsilon}^{s}(x)\right)=0$ for $m$-a.e. $x \in \Lambda$, then $m(N)=0$.

It is hard to locate a proof of Proposition 2.3 in the literature. In the more familiar case when $m$ is the measure of maximal entropy (the equilibrium state corresponding to $g=0$ ), then Proposition 2.3 was proved in [Si] for Anosov systems and [RS] for Axiom $A$ diffeomorphisms. For Axiom $A$ flows, the result for an arbitrary Hölder equilibrium state was proved by Haydn [Hy]. For completeness we give a sketch of the proof, modelled on [RS], as an appendix (10).

\section{Shifts of Finite type AND SOluble Lie Groups}

For shifts of finite type, Property (R) takes the form:

Property $\left(\mathbf{R}^{\prime}\right)$. If $f: \Sigma \rightarrow G$ is Hölder and $u$ is a measurable solution to the coboundary equation $f=u \sigma \cdot u^{-1}$ a.e., then $u$ has a Hölder continuous version.

Similarly we have the analogue of Property (PO):

Property $\left(\mathbf{P O}^{\prime}\right)$. If $f: \Sigma \rightarrow G$ is Hölder and $f^{n}(x)=e$ whenever $\sigma^{n} x=x$, then $f=u \sigma \cdot u^{-1}$ for a Hölder continuous $u$.

3.1. Lifting solutions. The following result will be used several times in the sequel.

Proposition 3.1. Suppose $G$ is a connected Lie group and $H$ is a normal subgroup. If Property $\left(R^{\prime}\right)$ holds for both $G / H$ and $H$, then Property $\left(R^{\prime}\right)$ holds for $G$.

Proof. Denote by $\pi$ the quotient homomorphism $G \rightarrow G / H$. Applying $\pi$ to the coboundary equation (1) gives the $G / H$-valued coboundary equation

$$
\pi(f)=\pi(u) \sigma \cdot \pi\left(u^{-1}\right)=\pi(u) \sigma \cdot \pi(u)^{-1} \text { a.e. }
$$

As Property $\left(\mathrm{R}^{\prime}\right)$ holds for $G / H$, we can find a Hölder function $v: \Sigma \rightarrow G / H$ such that $\pi(f)=v \sigma \cdot v^{-1}$ a.e. and $v=\pi(u)$ a.e.

As $\Sigma$ is totally disconnected, we can find a Hölder lift of $v$, i.e. a function $w$ : $\Sigma \rightarrow G$ such that $\pi(w)=v$.

Define $f^{\prime}=w^{-1} \sigma \cdot f \cdot w$. Then $f^{\prime}$ is Hölder; moreover $\pi\left(f^{\prime}\right)=v^{-1} \sigma \cdot \pi(f) \cdot v=e$ so that $f^{\prime}$ is $H$-valued. It is also clear that $w^{-1} \cdot v$ is a measurable function taking values in $H$ a.e. Hence $f^{\prime}=\left(w^{-1} u\right) \sigma \cdot\left(w^{-1} u\right)^{-1}$ is a coboundary equation taking values in $H$. As Property $\left(\mathrm{R}^{\prime}\right)$ holds for $H, w^{-1} u$ is equal a.e. to a Hölder function. Hence $u$ is equal a.e. to a Hölder function.

We have the following result for Property $\left(\mathrm{PO}^{\prime}\right)$.

Proposition 3.2. Suppose $G$ is a connected Lie group and $H$ is a normal subgroup. If Property $\left(P O^{\prime}\right)$ holds for both $G / H$ and $H$, then Property $\left(P O^{\prime}\right)$ holds for $G$.

Proof. The proof of Proposition 3.1 goes through, with Property $\left(\mathrm{PO}^{\prime}\right)$ replacing Property $\left(\mathrm{R}^{\prime}\right)$ at each occurrence. 
3.2. Property $\left(\mathbf{P O}^{\prime}\right)$ and Property $\left(\mathbf{R}^{\prime}\right)$ for soluble Lie groups. The results of $\$ 3.1$ allow us to prove the analogues of Theorems $\mathrm{A}$ and $\mathrm{B}$ for shifts of finite type and $G$ a connected soluble Lie group. Recall that a connected Lie group $G$ is said to be soluble if there are closed subgroups $G=G_{0}>G_{1}>\ldots>G_{n}=\{e\}$ such that $G_{i} / G_{i+1}$ is abelian, $0 \leq i \leq n-1$.

Proposition 3.3. Let $\sigma$ be a shift of finite type. If $G$ is a connected soluble Lie group, then both Property $\left(P O^{\prime}\right)$ and Property $\left(R^{\prime}\right)$ hold.

Proof. The proofs of the two cases are similar, so we only prove that Property $\left(\mathrm{R}^{\prime}\right)$ holds.

We use induction on the dimension of $G$. If $\operatorname{dim} G=1$, then $G$ is abelian and Property $\left(\mathrm{R}^{\prime}\right)$ is known to hold.

Suppose $\operatorname{dim} G=n>1$. If $G$ is abelian, then we are done. If not, then there exists a connected normal soluble subgroup $H$ such that $G / H$ is abelian and $\operatorname{dim} H<\operatorname{dim} G$. By the inductive hypothesis, Property $\left(\mathrm{R}^{\prime}\right)$ holds for both $H$ and $G / H$. By Proposition 3.1 Property $\left(\mathrm{R}^{\prime}\right)$ holds for $G$.

\section{INVARIANT GRAPHS OVER STABLE MANIFOLDS}

The following key result describes families of invariant graphs over stable and unstable manifolds. Define the skew product

$$
\begin{aligned}
\phi_{f} \quad: \quad & \Lambda \times G \rightarrow \Lambda \times G \\
& (x, y) \mapsto(\phi x, f(x) y) .
\end{aligned}
$$

Recall that we have defined $f^{n}(x)=f\left(\phi^{n-1} x\right) \cdots f(x)$.

Theorem 4.1. Let $\phi: \Lambda \rightarrow \Lambda$ be hyperbolic. Let $G$ be a connected Lie group and suppose $f: \Lambda \rightarrow G$ satisfies Hypothesis $(P H)$. Suppose $f \in C^{\theta}(\Lambda, G)$ for some $\theta>\theta_{\mathrm{PH}}$. Then for each $x \in \Lambda$, there exists a Hölder function $\gamma_{x}^{s}(\cdot): W^{s}(x) \cap \Lambda \rightarrow G$ such that

1. $\gamma_{x}^{s}(x)=e$,

2. on local stable manifolds, the Hölder constant of $\gamma_{x}^{s}(\cdot): W_{\varepsilon}^{s}(x) \cap \Lambda \rightarrow G$ is independent of $x$,

3. for $x^{\prime} \in W^{s}(x), \gamma_{x}^{s}\left(x^{\prime}\right)$ is defined by

$$
\gamma_{x}^{s}\left(x^{\prime}\right)=\lim _{n \rightarrow \infty} f^{n}\left(x^{\prime}\right)^{-1} f^{n}(x),
$$

4. if $W^{s}(x, y)$ denotes the graph of $\gamma_{x}^{s}(\cdot) y, y \in G$, then

$$
\phi_{f}\left(W^{s}(x, y)\right)=W^{s}\left(\phi_{f}(x, y)\right) .
$$

Similarly, we may define $\gamma_{x}^{u}$ on $W^{u}(x) \cap \Lambda$ by

$$
\gamma_{x}^{u}\left(x^{\prime}\right)=\lim _{n \rightarrow \infty} f^{-n}\left(x^{\prime}\right)^{-1} f^{-n}(x)
$$

where $f^{-n}(x)=f^{n}\left(\phi^{-n} x\right)^{-1}$.

Proof. Let $G$ be a connected Lie group. For $g \in G$, let $T_{g} G$ denote the tangent space at $g$ and identify $T_{e} G$ with $L(G)$, the Lie algebra of $G$. Let $l_{g}, r_{g}$ denote left, right multiplication by $g$, respectively. Let $d_{h} l_{g}, d_{h} r_{g}$ denote the derivatives of $l_{g}, r_{g}$ at $h$, respectively. The adjoint map Ad : $G \rightarrow \operatorname{Aut}(L(G))$ is defined by $\operatorname{Ad}(g) X=d_{g} r_{g^{-1}} d_{e} l_{g} X$ for $X \in T_{e} G$, that is, $\operatorname{Ad}(g)$ is the derivative of conjugation by $g$. 
Let $\|\cdot\|_{e}$ be a norm on $T_{e} G$. Define a Finsler norm by $\|X\|_{g}=\left\|d_{g} r_{g^{-1}} X\right\|_{e}$ for $X \in T_{g} G$. This defines a metric $\rho$ on $G$ and it is easy to see that $\rho$ is right-invariant: $\rho\left(g h, g^{\prime} h\right)=\rho\left(g, g^{\prime}\right)$ for all $g, g^{\prime}, h \in G$. Note that for $X \in T_{g} G$ we have

$$
\begin{aligned}
\left\|d_{g} l_{h} X\right\|_{h g} & =\left\|d_{h g} r_{(h g)^{-1}} d_{g} l_{h} X\right\|_{e} \\
& =\left\|d_{h} r_{h^{-1}} d_{e} l_{h} d_{g} r_{g^{-1}} X\right\|_{e} \\
& =\left\|\operatorname{Ad}(h) d_{g} r_{g^{-1}} X\right\|_{e}
\end{aligned}
$$

so that $\rho\left(h g, h g^{\prime}\right) \leq\|\operatorname{Ad}(h)\| \rho\left(g, g^{\prime}\right)$.

Recall from $\S \mathbb{1}$ the definition of $\mu_{s}$. By the assumption on $\theta$, choose $\delta>0$ such that $\lambda_{s}^{\theta}<\mu_{s}-\delta$. Then

$$
\sup _{x^{\prime}}\left\|\operatorname{Ad} f^{n}\left(x^{\prime}\right)^{-1}\right\| \leq\left(\mu_{s}-\delta\right)^{-n}
$$

provided $n$ is sufficiently large.

For each $x \in \Lambda$ and $x^{\prime} \in W^{s}(x) \cap \Lambda$, define

$$
\gamma_{x}^{s, n}\left(x^{\prime}\right)=f^{n}\left(x^{\prime}\right)^{-1} f^{n}(x) .
$$

We claim that $\gamma_{x}^{s}\left(x^{\prime}\right)=\lim _{n \rightarrow \infty} \gamma_{x}^{s, n}\left(x^{\prime}\right)$ exists.

To see this, note that by the right invariance of $\rho$ we have for large $n$

$$
\begin{aligned}
& \rho\left(\gamma_{x}^{s, n+1}\left(x^{\prime}\right), \gamma_{x}^{s, n}\left(x^{\prime}\right)\right) \\
& \quad=\rho\left(f^{n}\left(x^{\prime}\right)^{-1} f\left(\phi^{n} x^{\prime}\right)^{-1} f\left(\phi^{n} x\right) f^{n}(x), f^{n}\left(x^{\prime}\right)^{-1} f\left(\phi^{n} x\right)^{-1} f\left(\phi^{n} x\right) f^{n}(x)\right) \\
& \quad \leq\left\|\operatorname{Ad}\left(f^{n}\left(x^{\prime}\right)^{-1}\right)\right\| \rho\left(f\left(\phi^{n} x^{\prime}\right)^{-1}, f\left(\phi^{n} x\right)^{-1}\right) \\
& \quad \leq C\left(\left(\mu_{s}-\delta\right)^{-1} \lambda_{s}^{\theta}\right)^{n}
\end{aligned}
$$

where the constant is independent of $n$. Hence $\gamma_{x}^{s, n}\left(x^{\prime}\right)$ is Cauchy and therefore converges.

We now show that $\gamma_{x}^{s, n}\left(x^{\prime}\right): W_{\varepsilon}^{s}(x) \cap \Lambda \rightarrow G$ is uniformly Hölder in $x$. Let $x^{\prime}, x^{\prime \prime} \in W_{\varepsilon}^{s}(x) \cap \Lambda$. Then

$$
\begin{aligned}
& \rho\left(\gamma_{x}^{s, n}\left(x^{\prime}\right), \gamma_{x}^{s, n}\left(x^{\prime \prime}\right)\right) \\
& \quad=\rho\left(f^{n}\left(x^{\prime}\right)^{-1}, f^{n}\left(x^{\prime \prime}\right)^{-1}\right) \\
& \leq \sum_{j=0}^{n-1} \rho\left(f^{j}\left(x^{\prime}\right)^{-1} f\left(\phi^{j} x^{\prime}\right)^{-1} f^{n-j-1}\left(\phi^{j+1} x^{\prime \prime}\right)^{-1},\right. \\
& \left.\quad \leq f_{j=0}^{j}\left(x^{\prime}\right)^{-1} f\left(\phi^{j} x^{\prime \prime}\right)^{-1} f^{n-j-1}\left(\phi^{j+1} x^{\prime \prime}\right)^{-1}\right) \\
& \leq \sum_{j=0}^{n-1}\left\|\operatorname{Ad}\left(f^{j}\left(x^{\prime}\right)^{-1}\right)\right\| \rho\left(f\left(\phi^{j} x^{\prime}\right)^{-1}, f\left(\phi^{j} x^{\prime \prime}\right)^{-1}\right)
\end{aligned}
$$

As $\left\|\operatorname{Ad}\left(f^{j}\left(x^{\prime}\right)^{-1}\right)\right\| \leq C(f, \delta)\left(\mu_{s}-\delta\right)^{-j}$ for all $j \in \mathbb{N}$, we may let $n \rightarrow \infty$ to obtain $\rho\left(\gamma_{x}^{s}\left(x^{\prime}\right), \gamma_{x}^{s}\left(x^{\prime \prime}\right)\right) \leq C d\left(x^{\prime}, x^{\prime \prime}\right)^{\theta}$, for some constant $C>0$, independent of $x, x^{\prime}$.

The proof of 4 is similar.

\section{Property (R) and partial hyperbolicity}

We can now prove Property $(\mathrm{R})$ for Hölder functions $f$ satisfying the partial hyperbolicity hypothesis. We restate Theorem A. 
Theorem 5.1. Let $\phi: \Lambda \rightarrow \Lambda$ be hyperbolic, equipped with a Hölder equilibrium state $m$. Let $G$ be a connected Lie group. Suppose $f: \Lambda \rightarrow G$ satisfies Hypothesis $(P H)$ and $f \in C^{\theta}(\Lambda, G)$ for some $\theta>\theta_{\mathrm{PH}}$. Then Property $(R)$ holds.

Proof. Suppose $f$ satisfies the hypotheses in the statement of the theorem, and that $f=u \phi \cdot u^{-1}$ a.e. for some measurable $u$.

Let $X \subset \Lambda$ denote the full measure set on which $f(x)=u(\phi x) u(x)^{-1}$. By considering the full measure set $\bigcap_{n \in \mathbb{Z}} \phi^{-n} X$, there is no loss in assuming that $X$ is $\phi$-invariant.

By Proposition 2.3 we can write

$$
f^{n}\left(x^{\prime}\right)^{-1} f^{n}(x)=u\left(x^{\prime}\right) u\left(\phi^{n} x^{\prime}\right)^{-1} u\left(\phi^{n} x\right) u(x)^{-1}
$$

for $m$-a.e. $x \in \Lambda$ and $m_{x}^{s}$-a.e. $x^{\prime} \in W^{s}(x)$.

By Lusin's theorem ([피,$\S 55]$, for example), choose a set $U \subset X$ with $\mu(U)>1 / 2$ such that $\left.u\right|_{U}$ is uniformly continuous.

As $\mu(U)>1 / 2$, by the Ergodic Theorem for almost all $x, x^{\prime}$ there is a positive density of times $n_{i} \rightarrow \infty$ such that $\phi^{n_{i}} x, \phi^{n_{i}} x^{\prime} \in U$. In particular, if we let $n \rightarrow \infty$ through this subsequence in (3) we see that

$$
\lim _{n_{i} \rightarrow \infty} f^{n_{i}}\left(x^{\prime}\right)^{-1} f^{n_{i}}(x)=u\left(x^{\prime}\right) u(x)^{-1}
$$

for $m$-a.e. $x \in \Lambda$ and $m_{x}^{s}$-a.e. $x^{\prime} \in W^{s}(x)$.

Hence $u\left(x^{\prime}\right)=\gamma_{x}^{s}\left(x^{\prime}\right) u(x) m_{x}^{s}$-a.e. so that $u$ is equal almost everywhere to a uniformly Hölder continuous function along almost every local stable manifold. Similarly, $u$ is equal almost everywhere to a uniformly Hölder continuous function along almost every local unstable manifold.

By Theorem 4.1 and Proposition 19.1.1 of $[\mathrm{KH}]$, this proves the result.

\section{Property (PO) and Centre bunching}

We now prove Property (PO) for Hölder functions $f$ satisfying the centre bunching hypothesis. We restate Theorem B.

Theorem 6.1. Let $\phi: \Lambda \rightarrow \Lambda$ be hyperbolic and let $G$ be a connected Lie group. Suppose $f: \Lambda \rightarrow G$ satisfies Hypothesis $(C B)$ and $f \in C^{\theta}(\Lambda, G)$ for some $\theta>\theta_{\mathrm{CB}}$. Then Property (PO) holds.

We give the proof as a series of lemmas. Throughout, we assume that $f: \Lambda \rightarrow G$ is such that $f^{n}(x)=e$ whenever $\phi^{n} x=x$. For convenience, we assume that $z=\phi z$ is a fixed point of $\phi$ so that (in the notation of the proof of Theorem 4.1) $\gamma_{z}^{s, n}(x)=f^{n}(x)^{-1}, \gamma_{z}^{u, n}(x)=f^{-n}(x)^{-1}=f^{n}\left(\phi^{-n} x\right)\left(\right.$ as $f^{n}(z)=e$ for all $\left.n\right)$.

Lemma 6.2. For $x \in W(z)$, the homoclinic set through $z$, we have $\gamma_{z}^{s}(x)=\gamma_{z}^{u}(x)$.

Proof. Let $\nu=\max \left\{\lambda_{s}, \lambda_{u}^{-1}\right\}$. Let $x \in W(z)$ and choose $n>0$ sufficiently large so that $\phi^{-n} x, \phi^{n} x$ are close enough to apply Theorem 2.1] Hence we have a periodic point $w=\phi^{2 n} w$ such that

$$
\begin{aligned}
d\left(\phi^{j} x, \phi^{j} w\right) & \leq C \nu^{n-j} d\left(\phi^{-n} x, \phi^{n} x\right), \\
d\left(\phi^{-j} x, \phi^{-j} w\right) & \leq C \nu^{n-j} d\left(\phi^{-n} x, \phi^{n} x\right),
\end{aligned}
$$


for $j=0, \ldots, n$. By Hypothesis (CB) and the assumption on $\theta$, we can choose $\delta>0$ such that $\left(\mu_{u}+\delta\right) \nu^{\theta}<1$. We estimate

$$
\begin{aligned}
& \rho\left(\gamma_{z}^{s, n}(x)^{-1}, f^{n}(w)\right) \\
& \quad=\rho\left(f\left(\phi^{n-1} x\right) \cdots f(x), f\left(\phi^{n-1} w\right) \cdots f(w)\right) \\
& \leq \sum_{j=0}^{n-1} \rho\left(f^{n-j-1}\left(\phi^{j+1} x\right) f\left(\phi^{j} x\right) f^{j}(w), f^{n-j-1}\left(\phi^{j+1} x\right) f\left(\phi^{j} w\right) f^{j}(w)\right) \\
& \quad \leq \sum_{j=0}^{n-1}\left\|\operatorname{Ad}\left(f^{n-j-1}\left(\phi^{j+1} x\right)\right)\right\| \rho\left(f\left(\phi^{j} x\right), f\left(\phi^{j} w\right)\right) \\
& \leq C \sum_{j=0}^{n-1}\left(\mu_{u}+\delta\right)^{n-j} \nu^{\theta(n-j)} d\left(\phi^{-n} x, \phi^{n} x\right)^{\theta} \\
& \leq C d\left(\phi^{-n} x, \phi^{n} x\right)^{\theta}
\end{aligned}
$$

for some constants $C$ independent of $n$ and $x$. Similarly, we have

$$
\rho\left(\gamma_{z}^{u, n}(x)^{-1}, f^{n}\left(\phi^{-n} w\right)^{-1}\right) \leq C d\left(\phi^{-n} x, \phi^{n} x\right)^{\theta} .
$$

As $\phi^{2 n} w=w$, we have $f^{2 n}(w)=e$ so that $f^{n}\left(\phi^{-n} w\right)^{-1}=f^{n}(w)$. Hence,

$$
\rho\left(\gamma_{z}^{s, n}(x)^{-1}, \gamma_{z}^{u, n}(x)^{-1}\right) \leq C d\left(\phi^{-n} x, \phi^{n} x\right)^{\theta} .
$$

The lemma follows by letting $n \rightarrow \infty$.

Define $u: W(z) \rightarrow G$ by $u(x)=\gamma_{z}^{s}(x)=\gamma_{z}^{u}(x)$. Then

$$
\begin{aligned}
u(\phi x) & =\lim _{n \rightarrow \infty} f^{n}(\phi x)^{-1} \\
& =\lim _{n \rightarrow \infty} f(x) f^{n+1}(x)^{-1} \\
& =f(x) u(x)
\end{aligned}
$$

so that $u$ is a solution to the coboundary equation $f=u \phi \cdot u^{-1}$ on the dense set $W(z)$. We show that $u$ extends to a Hölder function defined on $\Lambda$. It is sufficient to show that $u$ is uniformly Hölder on $W(z)$.

Lemma 6.3. The function $u: W(z) \rightarrow G$ is uniformly Hölder.

Proof. Let $x, y \in W(z)$ be sufficiently close so that $w=\langle y, x\rangle$ is defined. Clearly $w \in W(z)$. Observe that

$$
\begin{aligned}
& \rho\left(\gamma_{z}^{s}(x), \gamma_{z}^{s}(w)\right) \\
& \quad=\lim _{n \rightarrow \infty} \rho\left(f(x)^{-1} \cdots f\left(\phi^{n-1} x\right)^{-1}, f(w)^{-1} \cdots f\left(\phi^{n-1} w\right)^{-1}\right) \\
& \leq \lim _{n \rightarrow \infty} \sum_{j=0}^{n-1} \rho\left(f^{j}(x)^{-1} f\left(\phi^{j} x\right)^{-1} f^{n-j-1}\left(\phi^{j+1} w\right)^{-1},\right. \\
& \quad \leq \lim _{n \rightarrow \infty} \sum_{j=0}^{n-1} \| \operatorname{Ad}\left(f^{j}(x)^{-1} f\left(\phi^{j} w\right)^{-1} f^{n-j-1}\left(\phi^{j+1} w\right)^{-1}\right) \\
& \quad \leq \lim _{n \rightarrow \infty} C \sum_{j=0}^{n-1}\left(\left(\phi^{j} x\right)^{-1}, f\left(\phi^{j} w\right)^{-1}\right)
\end{aligned}
$$


for some constant $C$ independent of $x, w$. It follows that

$$
\rho\left(\gamma_{z}^{s}(x), \gamma_{z}^{s}(w)\right) \leq C d(x, w)^{\theta} .
$$

Similarly, $\rho\left(\gamma_{z}^{u}(y), \gamma_{z}^{u}(w)\right) \leq C d(y, w)^{\theta}$. Hence

$$
\rho(u(x), u(y)) \leq C\left(d(x, w)^{\theta}+d(y, w)^{\theta}\right) \leq C d(x, y)^{\theta} .
$$

This proves Theorem 6.1 under the additional hypothesis that there exists a fixed point $z$. If no such point exists, then repeat the above construction with a periodic $p$ point, taking the limits through multiples of $p$. This gives a Hölder solution $u$ to $f^{p}=u \phi^{p} \cdot u^{-1}$. Using a period $q$ point, we have a Hölder solution $v$ to $f^{q}=v \phi^{q} \cdot v^{-1}$. By the transitivity of $\phi^{p q}$, we may take $v=u$. Choose $p, q$ to be coprime. Then for suitable integers $k, l$ we have $f(x)=f^{k p+l q}(x)=$ $f^{k p}\left(\phi^{l q} x\right) f^{l q}(x)=u\left(\phi^{k p+l q} x\right) u(x)^{-1}=u(\phi x) u(x)^{-1}$.

\section{Proofs of MAin Results}

We show how to combine the results of $\S \$ 3,56$ to obtain a large class of functions for which Property (R) and Property (PO) hold.

The following was used in [NP for linear Lie groups. The proof closely follows Livšic's original argument and we include it for completeness. This result applies without requiring the partial hyperbolicity assumption.

Proposition 7.1. Let $G$ be a connected Lie group and $\phi: \Lambda \rightarrow \Lambda$ a hyperbolic diffeomorphism equipped with a Hölder equilibrium state. If $f: \Lambda \rightarrow G$ is Hölder and satisfies $f=u \phi \cdot u^{-1}$ a.e. where both $u$ and $u^{-1}$ are essentially bounded, then $u$ has a Hölder continuous version.

Proof. Let $\rho$ denote a right-invariant metric on $G$.

Suppose $u, u^{-1}$ are bounded on a set of full measure $X$. By replacing $X$ by $\bigcap_{n \in \mathbb{Z}} \phi^{-n} X$ there is no loss in assuming that $X$ is $\phi$-invariant. Note that $\{u(x)$, $\left.u(x)^{-1} \mid x \in X\right\}$ has compact closure. Hence $\left\{f^{n}(x) \mid n \in \mathbb{Z}, x \in \Lambda\right\}$ has compact closure. In particular,

$$
D=\sup _{x \in X, n \in \mathbb{Z}}\left\{\left\|\operatorname{Ad}\left(f^{n}(x)\right)\right\|\right\}<\infty
$$

and we have $\rho\left(f^{n}(x) g, f^{n}(x) g^{\prime}\right) \leq D \rho\left(g, g^{\prime}\right)$ for all $n \in \mathbb{Z}, x \in \Lambda$ and $g, g^{\prime} \in G$.

By Lusin's theorem, choose a set $U \subset X$ of measure $\mu(U)>1 / 2$ such that $u,\left.u^{-1}\right|_{U}$ are uniformly continuous.

Let $x \in X$ be a point at which $f(x)=u(\phi x) u(x)^{-1}$. By Proposition [2.3 $f(y)=u(\phi y) u(y)^{-1}$ for almost all $y \in W^{u}(x)$ with respect to the induced measure on $W^{u}(x)$. Let $y \in W_{\varepsilon}^{u}(x)$ be such a point and let $x_{n}=\phi^{-n}(x), y_{n}=\phi^{-n}(y)$. Then $d\left(x_{n}, y_{n}\right) \leq C \lambda_{u}^{-n} d(x, y)$.

We have $u(x)=f^{n}\left(x_{n}\right) u\left(x_{n}\right)$. Hence,

$$
\begin{aligned}
\rho(u(x), u(y)) & =\rho\left(f^{n}\left(x_{n}\right) u\left(x_{n}\right), f^{n}\left(y_{n}\right) u\left(y_{n}\right)\right) \\
& \leq \rho\left(f^{n}\left(x_{n}\right), f^{n}\left(y_{n}\right)\right)+D \rho\left(u\left(x_{n}\right), u\left(y_{n}\right)\right) .
\end{aligned}
$$


Now

$$
\begin{aligned}
& \rho\left(f^{n}\left(x_{n}\right), f^{n}\left(y_{n}\right)\right) \\
& \quad \leq \sum_{j=0}^{n-1} \rho\left(f^{n-j-1}\left(\phi^{j+1} x_{n}\right) f\left(\phi^{j} x_{n}\right) f^{j}\left(x_{n}\right), f^{n-j-1}\left(\phi^{j+1} x_{n}\right) f\left(\phi^{j} y_{n}\right) f^{j}\left(y_{n}\right)\right) \\
& \quad \leq D \sum_{j=0}^{n-1} \rho\left(f\left(\phi^{j} x_{n}\right), f\left(\phi^{j} y_{n}\right)\right) \\
& \leq C d(x, y)^{\theta},
\end{aligned}
$$

where $\theta$ is the Hölder exponent of $f$ and $C$ is a constant, independent of $x, y$.

As $\mu(U)>1 / 2$, we can find for almost all $x, y$, a sequence $n_{i} \rightarrow \infty$ such that $\left(x_{n_{i}}, y_{n_{i}}\right) \in U \times U$. By Proposition 2.3, we may assume $x, y$ belong to the same unstable manifold. As $u$ is uniformly continuous on $U$, we have $\rho\left(u\left(x_{n_{i}}\right), u\left(y_{n_{i}}\right)\right) \rightarrow$ 0 as $n_{i} \rightarrow \infty$.

Letting $n \rightarrow \infty$ through a subsequence in (4) we have

$$
\rho(u(x), u(y)) \leq C d(x, y)^{\theta}
$$

for $x, y$ belonging to the same local unstable manifold, where $C$ is a constant, independent of $x, y$. Hence $u$ has a uniformly Hölder version on almost all local unstable manifolds.

Similarly $u$ has a uniformly Hölder version on almost all local stable manifolds.

By [KH, Proposition 19.1.1], this proves the result.

We can use Proposition 2.2 to prove that Property (R) holds for an arbitrary connected Lie group and a large class of functions $f$. Recall that $R$ denotes the radical of $G$, namely the largest connected normal soluble Lie subgroup.

Theorem 7.2. Let $\phi: \Lambda \rightarrow \Lambda$ be a hyperbolic diffeomorphism equipped with a Hölder equilibrium state. Let $G$ be a connected Lie group. Let $f: \Lambda \rightarrow G$ be Hölder and suppose $\pi_{R} f: \Lambda \rightarrow G / R \in C^{\theta}(\Lambda, G / H)$ satisfies Hypothesis $(P H)$ and $\theta>\theta_{\mathrm{PH}}$. Then Property $(R)$ holds.

Proof. Suppose $f=u \phi \cdot u^{-1}$ a.e. for some measurable $u$. Let $\pi_{R}: G \rightarrow G / R$ be the quotient homomorphism. Then $\pi_{R}(f)=\pi_{R}(u \phi) \cdot \pi_{R}(u)^{-1}$ a.e. and this is a coboundary equation satisfying the hypotheses of Theorem [5.1. Hence $\pi_{R}(u)=v$ a.e. for some Hölder $v: \Lambda \rightarrow G / R$.

Let $\pi: \Sigma \rightarrow \Lambda$ be as in Proposition 2.2, Then $f \pi=(u \pi) \sigma \cdot(u \pi)^{-1}$ a.e. with respect to a Hölder equilibrium state for $\sigma: \Sigma \rightarrow \Sigma$. Moreover, $\pi_{R}(u \pi)=v \pi$ a.e. and $v \pi: \Sigma \rightarrow G / R$ is Hölder. Hence Property $\left(\mathrm{R}^{\prime}\right)$ holds for $f \pi$ and $G / R$. By Proposition 3.3. Property $\left(\mathrm{R}^{\prime}\right)$ holds for $R$. Hence by Proposition $3.1 f \pi=u^{\prime} \sigma \cdot u^{\prime-1}$ everywhere for some Hölder $u^{\prime}: \Sigma \rightarrow G$ such that $u^{\prime}=u \pi$ a.e.

As $\pi$ is one-to-one a.e., it follows from $u^{\prime}=u \pi$ a.e. and the continuity of $u^{\prime}$ that $u$ is essentially bounded. By Proposition $7.1 u$ has a Hölder version, and the Hölder exponent is the same as that of $f$.

Essentially the same proof, using Proposition 3.2, gives the following:

Theorem 7.3. Let $\phi: \Lambda \rightarrow \Lambda$ be a hyperbolic diffeomorphism and let $G$ be a connected Lie group. Let $f: \Lambda \rightarrow G$ be Hölder and suppose $\pi_{R} f: \Lambda \rightarrow G / R \in$ $C^{\theta}(\Lambda, G / H)$ satisfies Hypothesis $(C B)$ and $\theta>\theta_{\mathrm{CB}}$. Then Property (PO) holds. 
We can also prove analogues of Theorems $7.2,7.3$ for shifts of finite type. Recall from 92 that with respect to the metric $d^{\theta}$ on $\Sigma, \sigma$ contracts $W^{s}(x)$ by $\theta$ and expands $W^{u}(x)$ by $\theta^{-1}$. The proofs in $\S \$ 5$ go through provided $f \in F_{\theta}(\Sigma, G)$ is such that $\theta<\theta_{\text {crit }}=\min \left\{\mu_{s}, \mu_{u}^{-1}\right\}$. Hence

Theorem 7.4. Let $\sigma: \Sigma \rightarrow \Sigma$ be a shift of finite type equipped with a Hölder equilibrium state. Let $G$ be a connected Lie group. If $f \in F_{\theta}(\Sigma, G)$ and $\theta<\theta_{\text {crit }}$, then Property $(R)$ holds.

Theorem 7.5. Let $\sigma: \Sigma \rightarrow \Sigma$ be a shift of finite type and let $G$ be a connected Lie group. If $f \in F_{\theta}(\Sigma, G)$ and $\theta<\theta_{\text {crit }}$, then Property (PO) holds.

\section{FLOWS}

We briefly explain how the results in this paper carry over to the case of continuous time.

A $C^{1}$ flow $\phi^{t}$ restricted to an invariant set $\Lambda$ is said to be hyperbolic if the Mather spectrum of the time-1 map is contained in $\left\{z \in \mathbb{C}|0<| z \mid<\lambda_{s}\right\} \cup$ $\left\{z \in \mathbb{C}\left|\lambda_{u}<\right| z \mid<\infty\right\} \cup\{z \in \mathbb{C}|| z \mid=1\}$ and the subspace of the tangent bundle of $\Lambda$ corresponding to the eigenvalue 1 is one-dimensional and spanned by the vector field generating the flow (we also assume that the periodic points are dense, there is a dense orbit and $\Lambda$ is locally maximal). Equilibrium states for hyperbolic flows can be defined in a similar way as for diffeomorphisms.

Let $G$ be a connected Lie group. A continuous map $\mathcal{F}: \Lambda \times \mathbb{R} \rightarrow G$ is a cocycle if $\mathcal{F}^{t+s}(x)=\mathcal{F}^{t}\left(\phi^{s} x\right) \mathcal{F}^{s}(x)$. A cocycle determines and is determined by the Lie algebra valued function

$$
f(x)=\lim _{t \rightarrow 0} \frac{1}{t} \exp ^{-1} \mathcal{F}^{t}(x) .
$$

We say that $\mathcal{F}$ is Hölder or $C^{r}$ if $f$ is Hölder or $C^{r}$, respectively.

Property (R) takes the form:

Property $(\mathbf{R})$ for hyperbolic flows. If $\mathcal{F}$ is a Hölder cocycle and $u$ is a measurable solution to the coboundary equation

$$
\mathcal{F}^{t}(x)=u\left(\phi^{t} x\right) u(x)^{-1} \text { a.e. },
$$

then $u$ has a Hölder continuous version.

Hypothesis $(\mathrm{PH})$ in continuous time takes the form:

Hypothesis (PH) for hyperbolic flows. Let $\phi^{t}$ be a hyperbolic flow and suppose the Mather spectrum of $\phi^{1}$ is contained in the annuli $\left\{z|0<| z \mid<\lambda_{s}\right\} \cup\{z \mid$ $\left.\lambda_{u}<|z|<\infty\right\}$ and the unit circle. A cocycle $\mathcal{F}$ satisfies Hypothesis (PH) if $\lambda_{s}<\mu_{s} \leq 1 \leq \mu_{u}<\lambda_{u}$ where

$$
\begin{aligned}
& \mu_{s}=\lim _{t \rightarrow \infty} \sup _{x \in \Lambda}\left\|\operatorname{Ad} \mathcal{F}^{t}(x)\right\|^{1 / t}, \\
& \mu_{u}=\lim _{t \rightarrow \infty} \sup _{x \in \Lambda}\left\|\operatorname{Ad} \mathcal{F}^{t}(x)^{-1}\right\|^{-1 / t} .
\end{aligned}
$$

Similarly, we have Property (PO) for hyperbolic flows:

Property (PO) for hyperbolic flows. If $\mathcal{F}$ is a Hölder cocycle such that $\mathcal{F}^{T}(x)$ $=e$ whenever $\phi^{T} x=x$ then $\mathcal{F}^{t}(x)=u\left(\phi^{t} x\right) u(x)^{-1}$ for some Hölder $u: \Lambda \rightarrow G$.

The centre bunching hypothesis remains the same: 
Hypothesis (CB) for hyperbolic flows. A cocycle $\mathcal{F}$ satisfies Hypothesis (CB) if it satisfies Hypothesis (PH), $\lambda_{s}<\mu_{u}^{-1}$ and $\mu_{s}^{-1}<\lambda_{u}$.

The analogues of Theorems 7.2 [7.3 are as follows:

Theorem 8.1. Let $\phi^{t}: \Lambda \rightarrow \Lambda$ be a hyperbolic flow, equipped with a Hölder equilibrium state. Let $G$ be a connected Lie group. Let $\mathcal{F}$ be a Hölder cocycle and suppose $\pi_{R} \mathcal{F}$ is a $G / R$-valued cocycle satisfying Hypothesis (PH) and the Hölder exponent of $\pi_{R} \mathcal{F}$ is greater than $\theta_{\mathrm{PH}}$. Then Property $(R)$ holds.

Theorem 8.2. Let $\phi^{t}: \Lambda \rightarrow \Lambda$ be a hyperbolic flow and let $G$ be a connected Lie group. Let $\mathcal{F}$ be a Hölder cocycle and suppose $\pi_{R} \mathcal{F}$ is a $G / R$-valued cocycle satisfying Hypothesis $(C B)$ and the Hölder exponent of $\pi_{R} \mathcal{F}$ is greater than $\theta_{\mathrm{CB}}$. Then Property $(R)$ holds.

The proofs follow the same general arguments as above and we merely sketch the necessary modifications.

The proofs of Theorem 5.1 and Proposition 7.1 generalise to show that, under Hypothesis (PH), a solution $u$ to (5) has a Hölder continuous version along almost every stable and unstable manifold. As $u\left(\phi^{t} x\right)=\mathcal{F}^{t}(x) u(x)$ a.e., $u$ has a Hölder version along orbits. By repeated use of [KH, Proposition 19.1.1], this is sufficient to conclude that $u$ has a Hölder version.

Consider Property (PO). For hyperbolic flows, it is not in general true that $W(z)$ is dense for any $z \in \Lambda$ (this is the case if $\phi^{t}$ is a constant suspension of a hyperbolic diffeomorphism, for example). However, the cocycle equation $\mathcal{F}^{t}(x)=$ $u\left(\phi^{t} x\right) u(x)^{-1}$ is invariant under velocity changes and we can always change velocity $\phi^{t}$ into a mixing flow. If $\phi^{t}$ is mixing, then $W(z)$ is dense for each $z \in \Lambda$. Under Hypothesis (CB), the proof of Theorem 6.1 generalises when $\phi^{t}$ is mixing.

The soluble case is slightly more involved. We first consider the case where $\phi^{t}$ is a suspension of a shift of finite type, so that $\Lambda=\Sigma \times[0,1] /(x, 1) \sim(\sigma x, 0)$ for a shift of finite type $\sigma$ on $\Sigma$. As in [W1, we first interpret (5) on a cross-section of the form $\Sigma \times\left\{t_{0}\right\}$. As this is zero-dimensional, the lifting arguments in the proof of Proposition 3.1 work. A continuous solution can then be constructed on $\Lambda$, as in [W1. Hence the analogue of Property $\left(\mathrm{R}^{\prime}\right)$ holds.

For Property (PO) in the soluble case, we again first consider a suspension of a shift of finite type. By reparametrising time, there is no loss in considering a constant suspension. In particular, the cross-section $\Sigma \times\{0\}$ is invariant under $\sigma$ and has the same periodic orbits as the suspended flow. This allows us to construct a continuous solution to the coboundary equation, first on $\Sigma \times\{0\}$ and then on $\Lambda$. This proves the analogue of Property $\left(\mathrm{PO}^{\prime}\right)$.

The two cases can be combined as in \$7] using Markov sections in place of Markov partitions.

\section{Concluding Remarks}

More generally, we could consider the equation

$$
f(x)=u(\phi x) g(x) u(x)^{-1} \text { a.e. }
$$

where $f, g: \Lambda \rightarrow G$ are Hölder and $u$ is measurable. When the group $G$ is compact (or, more generally, if $f, g$ satisfy Schmidt's notion of bounded distortion) it is again true that $u$ must have a Hölder continuous version [PP2, Sc]. 
When $G$ is non-compact, the situation is less clear. When $G$ is nilpotent, measurable solutions to (6) must be Hölder; simple modifications to the proofs of Proposition 3.1 and Proposition 3.3 prove this. We shall give an example of a soluble Lie group for which ([6) has a measurable solution, but no continuous solution.

Take $G$ to be the connected soluble linear Lie group

$$
G=\left\{\left(\begin{array}{cc}
a_{1} & a_{2} \\
0 & 1
\end{array}\right) \mid a_{1}>0, a_{2} \in \mathbb{R}\right\} .
$$

Let

$$
f=\left(\begin{array}{cc}
f_{1} & f_{2} \\
0 & 1
\end{array}\right), g=\left(\begin{array}{cc}
g_{1} & g_{2} \\
0 & 1
\end{array}\right), u=\left(\begin{array}{cc}
u_{1} & u_{2} \\
0 & 1
\end{array}\right) .
$$

We shall show that for an appropriate choice of $f$ and $g$, (6) has a measurable solution $u$, but no continuous solution.

Let $x_{0}=\phi\left(x_{0}\right)$ be a fixed point. Choose $f$ and $g$ such that

1. $f_{1}=g_{1}$;

2. $f_{1}\left(x_{0}\right)=1$ and $f_{2}\left(x_{0}\right) \neq g_{2}\left(x_{0}\right)$;

3. for almost all $x \in \Lambda$,

$$
\lim _{n \rightarrow \infty}\left(f_{1}(x) f_{1}(\phi x) \cdots f_{1}\left(\phi^{n-1} x\right)\right)^{1 / n} \rightarrow \alpha<1
$$

(the limit exists and is constant a.e. by the ergodicity of $\phi$ ). The first assumption allows us to take $u_{1}=1$. Equation (6) then reduces to

$$
f_{2}(x)=u_{2}(\phi x)+g_{2}(x)-f_{1}(x) u_{2}(x) \text { a.e.. }
$$

If $u_{2}$ were to have a continuous version, then (7) would hold everywhere. However, by setting $x=x_{0}$, this contradicts the second assumption.

It remains to check that (7) admits a measurable solution $u_{2}$. Let $\varepsilon>0$ be small. The set

$$
X=\left\{x \in \Lambda \mid f_{1}(x) f_{1}(\phi x) \cdots f_{1}\left(\phi^{n-1} x\right)<c(x)(\alpha+\varepsilon)^{n}\right\}
$$

has measure one (here $c(x)$ is a constant depending on $x$, but independent of $n$ ). By replacing $X$ by $\bigcap_{n \in \mathbb{Z}} \phi^{-n} X$ we may assume that $X$ is $\phi$-invariant. Define for each $n>0$

$$
F^{n}(x)=f_{2}(x)+f_{1}(x) f_{2}(\phi x)+\cdots+f_{1}(x) \cdots f_{1}\left(\phi^{n-1} x\right) f_{2}\left(\phi^{n} x\right) .
$$

Then

$$
\begin{aligned}
& F^{n}(x)=F^{n-1}(x)+f_{1}(x) \cdots f_{1}\left(\phi^{n-1} x\right) f_{2}\left(\phi^{n} x\right), \\
& F^{n}(x)=f_{2}(x)+f_{1}(x) F^{n-1}(\phi x) .
\end{aligned}
$$

Fix $x \in X$. From (8) it follows that

$$
\left|F^{n+j}(x)-F^{n+j-1}(x)\right|<\left\|f_{2}\right\|_{\infty} c(x)(\alpha+\varepsilon)^{n+j} .
$$

Hence,

$$
\begin{aligned}
\left|F^{n+m}(x)-F^{n}(x)\right| & \leq \sum_{j=1}^{m}\left|F^{n+j}(x)-F^{n+j-1}(x)\right| \\
& \leq c(x)(\alpha+\varepsilon)^{n} \sum_{j=0}^{\infty}(\alpha+\varepsilon)^{j} \\
& \leq C(x)(\alpha+\varepsilon)^{n}
\end{aligned}
$$


and it follows that, for each $x, F^{n}(x)$ is Cauchy. Call the limit $v(x)$; this is a measurable function. Letting $n \rightarrow \infty$ in (9) we have $f_{2}(x)=v(x)-f_{1}(x) v(\phi x)$. Then $u_{2}=v \phi^{-1}$ is a measurable solution to (7).

One can also study smooth versions of Property $(\mathrm{R})$ : if $\phi$ is a smooth $\left(C^{\infty}\right)$ Anosov diffeomorphism and $f$ is a smooth function such that $f=u \phi \cdot u^{-1}$ for some continuous $u$, then must $u$ itself be smooth? This, and the related $C^{r}$ problem, has been well-studied and we refer to [NT] for details and references.

\section{Appendix: Proof of Proposition 2.3}

Proposition 2.3 was proved for the equilibrium state corresponding to the function $g=0$ (namely, the measure of maximal entropy) in $[\mathrm{RS}$. Here we sketch the modifications needed in their argument to prove Proposition 2.3] for an arbitrary Hölder equilibrium state.

Let $m$ be the equilibrium state for $\phi$ corresponding to $g$. Then $m=\pi_{*} \tilde{m}$ where $\pi: \Sigma \rightarrow \Lambda$ is as in Proposition 2.2 and $\tilde{m}$ is the equilibrium state for $\sigma$ corresponding to the Hölder function $\tilde{g}=g \pi$. We first prove the Proposition in the case of a shift of finite type, and we drop the tildes for simplicity.

Define $\Sigma^{+}=\left\{x=\left(x_{n}\right)_{n=0}^{\infty} \mid A_{x_{n} x_{n+1}}=1\right.$ for $\left.n \geq 0\right\}$ to be the one-sided shift space, and $\sigma: \Sigma^{+} \rightarrow \Sigma^{+}:(\sigma x)_{n}=x_{n+1}$ to be the one-sided shift. If $x \in \Sigma$, then we can regard $W_{\varepsilon}^{u}(x)$ as a subspace of $\Sigma^{+}$, namely those sequences $\left(y_{n}\right)_{n=0}^{\infty}$ for which $y_{0}=x_{0}$.

Let $g: \Sigma \rightarrow \mathbb{R} \in F_{\theta}(\Sigma, \mathbb{R})$. By subtracting $P(g)$ we may assume that $P(g)=0$. Moreover, we may find $v_{u} \in F_{\theta^{\frac{1}{2}}}(\Sigma, \mathbb{R})$ such that

1. $g_{u}=g+v_{u}-v_{u} \sigma \in F_{\theta^{\frac{1}{2}}}(\Sigma, \mathbb{R})$ depends only on future coordinates (i.e. $g_{u}(x)=g_{u}(y)$ if $x_{i}=y_{i}$ for $\left.i \geq 0\right)$, and so can be regarded as a function $g_{u}: \Sigma^{+} \rightarrow \mathbb{R}$

2. $g_{u}$ is normalised: $\sum_{y \in \Sigma^{+}: \sigma y=x} e^{g_{u}(y)}=1$ for each $x \in \Sigma^{+}$.

Recall the transfer operator

$$
L_{g_{u}}: F_{\theta^{\frac{1}{2}}}\left(\Sigma^{+}, \mathbb{R}\right) \rightarrow F_{\theta^{\frac{1}{2}}}\left(\Sigma^{+}, \mathbb{R}\right):\left(L_{g_{u}} w\right)(x)=\sum_{y: \sigma y=x} e^{g_{u}(y)} w(y) .
$$

Then the spectral radius of $L_{g_{u}}$ is $1, L_{g_{u}} 1=1$ and 1 is a simple isolated eigenvalue. The equilibrium state $m$ of $g$ (which is also the equilibrium state of $g_{u}$ ) is characterised by $L_{g_{u}}^{*} m=m$.

Define the measure $m_{x}^{u}$ on $W_{\varepsilon}^{u}(x)$ by $\left(d m_{x}^{u} / d m\right)(\cdot)=e^{v_{u}(\langle x, \cdot\rangle)}$, regarding $W_{\varepsilon}^{u}(x)$ as a subset of $\Sigma^{+}$.

By [PP1, Prop. 3.2] $d \sigma^{*} m_{x}^{u}=e^{g(\sigma\langle x, \cdot\rangle)} d m_{\sigma x}^{u}$ and the family of measures have the claimed scaling properties on unstable manifolds.

One can easily check that $\left(d \rho_{x, x^{\prime}}^{*} m_{x}^{u} / d m_{x^{\prime}}^{u}\right)(\cdot)=e^{v_{u}(\langle x, \cdot\rangle)-v_{u}\left(\left\langle x^{\prime}, \cdot\right\rangle\right)}$. Define $g^{n}(x)$ $=g(x)+\cdots+g\left(\sigma^{n-1} x\right)$. Then

$$
\begin{aligned}
& v_{u}(\langle x, \cdot\rangle)-v_{u}\left(\left\langle x^{\prime}, \cdot\right\rangle\right) \\
& =g^{n}(\langle x, \cdot\rangle)-g^{n}\left(\left\langle x^{\prime}, \cdot\right\rangle\right)-g_{u}^{n}(\langle x, \cdot\rangle)+g_{u}^{n}\left(\left\langle x^{\prime}, \cdot\right\rangle\right) \\
& \quad+v_{u}\left(\sigma^{n}\langle x, \cdot\rangle\right)-v_{u}\left(\left\langle x^{\prime}, \cdot\right\rangle\right) .
\end{aligned}
$$

As $g_{u}$ depends only on future coordinates, the terms involving $g_{u}$ cancel. Letting $n \rightarrow \infty$ we see that

$$
\omega_{x, x^{\prime}}(y)=\lim _{n \rightarrow \infty} g^{n}(\langle x, \cdot\rangle)-g^{n}\left(\left\langle x^{\prime}, \cdot\right\rangle\right)=v_{u}(\langle x, \cdot\rangle)-v_{u}\left(\left\langle x^{\prime}, \cdot\right\rangle\right)
$$

is well-defined. Hence the measures $m_{x}^{u}$ transform as claimed under holonomy. 
Similarly, we can define measures $m_{x}^{s}$ on $W_{\varepsilon}^{s}(x)$ by regarding $W_{\varepsilon}^{s}(x)$ as a subset of $\Sigma^{-}=\left\{\left(x_{n}\right)_{n=-\infty}^{0} \mid A_{x_{n-1} x_{n}}=1\right.$ for $\left.n \leq 0\right\}$ and repeating the above construction using $\sigma^{-1}$ in place of $\sigma$ and $g_{s}$ in place of $g_{u}$, where $g_{s}=g+v_{s}-v_{s} \sigma$ depends only on the 'past'.

Using the product structure $\langle\cdot, \cdot \cdot\rangle$ it is easy to see that

$$
d\left(m_{x}^{s} \times m_{x}^{u}\right)=e^{v_{u}(\langle x, \cdot\rangle)-v_{s}(\langle\cdot, x\rangle)} d m
$$

Finally, we want to push down the measure $m_{x}^{s, u}$ from $\Sigma$ to $\Lambda$. Let $\mathcal{R}=\left\{R_{i}\right\}$ be a Markov partition such that $\operatorname{diam} R_{i}<\varepsilon$. Then $\pi: \Sigma \rightarrow \Lambda$ is given by $\pi\left(x_{n}\right)=\bigcap_{n \in \mathbb{Z}} \phi^{-n} R_{x_{n}}$. Let $x \in \Lambda$ and suppose $x \in R_{x_{0}}$. Choose $\left(x_{n}\right) \in \Sigma$ such that $\pi\left(x_{n}\right)=x$. We define the measure $m_{x}^{u}$ on $W_{\varepsilon}^{u}(x) \cap R_{x_{0}}$ by projecting the measure $m_{\left(x_{n}\right)}^{u}$ on $W_{\varepsilon}^{u}\left(\left(x_{n}\right)\right)$ constructed above. That this is well-defined follows from well-known properties of Markov partitions and is given in detail in RS.

\section{REFERENCES}

[A] D. V. Anosov, Geodesic Flows on Closed Riemannian Manifolds with Negative Curvature, Proc. Steklov Inst., vol. 90, Amer. Math. Soc., Prov., Rhode Island, 1969. MR 39:3527

[B] R. Bowen, Equilibrium States and the Ergodic Theory of Anosov Diffeomorphisms, Lecture Notes in Math., vol. 470, Springer-Verlag, Berlin, 1975. MR 56:1364

[BP] M. I. Brin and Ya. B. Pesin, Partially hyperbolic dynamical systems, Math. U.S.S.R., Izv. 8 (1974), 177-218.

[Hl] P. Halmos, Measure theory, Van Nostrand, New York, 1950. MR 11:504d

[Hy] N. Haydn, Canonical product structure of equilibrium states, Random. Comput. Dynam. 2 (1994), 79-96. MR 95c:58133

[KH] A. Katok and B. Hasselblatt, Introduction to the Modern Theory of Dynamical Systems, Encyclopedia of Math., vol. 54, Cambridge Univ. Press, Cambridge, 1995. MR 96c:58055

[L1] A. N. Livšic, Homology properties of Y-systems, Math. Notes 10 (1971), 758-763.

[L2] A. N. Livšic, Cohomology of dynamical systems, Math. U.S.S.R., Izv. 6 (1972), 1278-1301.

[NP] M. Nicol and M. Pollicott, Measurable cocycle rigidity for some non-compact groups, Bull. London. Math. Soc. 31 (1999), 592-600. MR 2000k:37004

[NT] V. Niţică and A. Török, Regularity of the coboundary for cohomologous cocycles, Ergod. Th. \& Dyn. Syst. 18 (1998), 1187-1209. MR 2000m:37030

[P] W. Parry, The Livšic periodic point theorem for two non-abelian cocycles, Ergod. Th. \& Dyn. Syst. 19 (1999), 687-701. MR 2000d:37019

[PP1] W. Parry and M. Pollicott, Zeta Functions and the Periodic Orbit Structure of Hyperbolic Dynamics, Astérique, vol. 187-188, Société Mathématique de France, 1990. MR 92f:58141

[PP2] W. Parry and M. Pollicott, The Livsic cocycle equation for compact Lie group extensions of hyperbolic systems, J. London Math. Soc. 56 (1997), 405-416. MR 99d:58109

[RS] D. Ruelle and D. Sullivan, Currents, flows and diffeomorphisms, Topology 14 (1975), 319-327. MR 54:3759

[Sc] K. Schmidt, Remarks on Livšic' theory for nonabelian cocycles, Ergod. Th. \& Dyn. Syst. 19 (1999), 703-721. MR 2000f:37036

[Si] Ya. G. Sinai, Markov partitions and C-diffeomorphisms, Funkts. Anal. Prilozh. 2 (1968), 64-89.

[W1] C. P. Walkden, Livšic theorems for hyperbolic flows, Trans. Amer. Math. Soc. 352 (2000), 1299-1313. MR 2000j:37036

[W2] C. P. Walkden, Livšic regularity theorems for twisted cocycle equations over hyperbolic systems, J. London Math. Soc. 61 (2000), 286-300. CMP 2000:09

Department of Mathematics, University of Manchester, Oxford Road, Manchester M13 9PL, U.K.

E-mail address: mp@ma.man.ac.uk

Department of Mathematics, University of Manchester, Oxford Road, Manchester M13 9PL, U.K.

E-mail address: cwalkden@ma.man.ac.uk 